Zamarra, J.; Guevara, J.; Ochoa, L. (2018). Lineamientos para elaborar una propuesta de servicios de aseguramiento: consideraciones y guía. Contaduría Universidad de Antioquia, 73, 103-129.

Doi: https://doi.org/10.17533/udea.rc.n73a05

\title{
Lineamientos para elaborar una propuesta de servicios de aseguramiento: consideraciones y guía
}

\author{
Julian Esteban Zamarra Londoño \\ julian.zamarra@udea.edu.co \\ Universidad de Antioquia
}

Jaime Alberto Guevara Sanabria

jalberto.guevara@udea.edu.co

Universidad de Antioquia

Lina María Ochoa Bustamante

linaochoab@hotmail.com

Grupo Old Mutual Colombia 
Lineamientos para elaborar una propuesta de servicios de aseguramiento: consideraciones y guía Resumen: En el desempeño profesional de los contadores públicos se encuentra la línea de auditoría, la cual en Colombia suele estar representada en la mayoría de los casos por la figura de la revisoría fiscal; sin embargo, existen otros campos de acción como lo son la del auditor externo que ofrece diferentes servicios bien sea de auditoría de estados financieros u otros servicios relacionados. Teniendo en consideración que la elección de las figuras del revisor fiscal o auditor externo en la mayoría de veces se llevan a cabo con una selección basada en la presentación de una propuesta de auditoría, el presente artículo expone diferentes consideraciones y una guía que contiene los elementos que debe contener una propuesta, permitiéndole a la entidad que hará la elección contar con elementos suficientes para tomar la mejor decisión en términos de calidad, y contar con el profesional idóneo y el más competente de aquellos que se postulen en el proceso de selección.

Palabras clave: Propuesta de servicios profesionales, revisor fiscal, auditor externo, dictamen de estados financieros, auditoría financiera, sistemas de control interno.

\section{Guidelines to create an assurance services proposal: considerations and guide}

Abstract: Auditing is one of the lines in the professional practice of public accountants, and this is in most cases represented in Colombia by the figure of the tax auditor. However, there are other work fields such as the external auditor, which offers several services, whether financial statements auditing or other related services. Taking into account that the choice between the tax auditor or external auditor is mostly made through a selection based on the presentation of an auditing proposal, the present paper sets forth different considerations and a guide with the elements that must be contained in such proposal, providing the entity with enough elements to make the best decision in terms of quality, and hire the most suitable and competent professional from those applying to the selection process.

Keywords: Professional services proposal, tax auditor, external auditor, financial statements report, financial auditing, internal control systems.

\section{Lignes directrices pour l'élaboration d'une proposition de services d'assurance : quelques considérations et guide}

Résumé : Le service d'audit fait partie de la pratique professionnelle des comptables publics. En Colombie, ce service est représenté - dans la plupart des cas- par la figure de expert-comptable. Néanmoins, il existe d'autres domaines d'action tels que l'auditeur externe, qui offre des services d'audit des états financiers ou d'autres services liés à ceux-ci. En général, le choix de l'expertcomptable ou d'auditeur externe est basé sur une proposition d'audit de la part des candidats. Ainsi, cet article expose quelques considérations et présente une guide contenant les éléments propres à une proposition, ce qui permet à l'établissement qui fera le choix du candidat de compter sur les éléments suffisants pour prendre la meilleure décision en termes de qualité, et de compter sur le professionnel idéal et le plus compétent parmi les candidats.

Mots-clés : proposition de services professionnels, expert-comptable, auditeur externe, rapport d'états financiers, contrôle financier, systèmes de contrôle interne.

\section{Parâmetros para elaborar uma proposta de serviços de asseguração: considerações e guia}

Resumo: No desempenho profissional dos contadores públicos encontra-se o eixo da auditoria, a qual na Colômbia geralmente está representada pela figura de "Revisoria Fiscal"; porém, existem outros campos de ação como o de "auditor externo" que oferece diferentes serviços, seja de auditoria dos estados financeiros ou outros serviços relacionados. Levando em consideração que a escolha das figuras de Revisor Fiscal ou auditor externo na maioria das vezes são efetuadas com uma seleção baseada na apresentação de uma proposta de auditoria, este artigo mostra diferentes considerações e uma guia que contém os elementos com que deve contar uma proposta, permitindo à entidade que fará a escolha ter elementos suficientes para tomar a melhor decisão em termos de qualidade e contar com o profissional idôneo e o más competente entre aqueles que se candidatarem ao processo de seleção.

Palavras chave: Proposta de serviços profissionais, revisor fiscal, auditor externo, ditame de estados financeiros, auditoria financeira, sistema de controle interno. 


\title{
Lineamientos para elaborar una propuesta de servicios de aseguramiento: consideraciones y guía
}

\author{
Julian Estaben Zamarra Londoño, Jaime Alberto Guevara Sanabria, Lina Ochoa \\ Doi: https://doi.org/10.17533/udea.rc.n73a05
}

Primera versión recibida en septiembre 2018 - Versión final aceptada en octubre 2018

\section{Introducción}

I a formación profesional de los contadores públicos en Colombia en la línea Lde auditoría por procesos, suele enfocarse en el conocimiento y desarrollo de competencias técnicas, tales como el entendimiento del control interno, la ejecución de las fases de auditoría aplicadas sobre los ciclos de negocio de las empresas, el estudio y cumplimiento de las normas locales e internacionales de auditoría, las responsabilidades y funciones del Revisor Fiscal en Colombia, entre otros. Sin embargo, se observan debilidades en la enseñanza profesional en lo referente a situaciones a las que se debe enfrentar un contador, cuando en su desarrollo profesional, decide desempeñarse como revisor fiscal y para ello, debe presentar una propuesta de servicios profesionales como insumo para su elección por parte de la entidad que requiere de este profesional. Es así como este artículo expone algunas consideraciones y desarrolla una guía útil para la elaboración de la mencionada propuesta.

El objetivo del presente artículo es brindar, a partir de la experiencia de los autores y diversos referentes bibliográficos, una guía a los estudiantes y profesionales de las ciencias contables, una primera articulación de los principales elementos que debe contener una propuesta de servicios profesionales de revisoría fiscal, auditoría externa y, en general, otros trabajos que impliquen aseguramiento por parte del profesional contable.

Finalmente, con este escrito se pretende contribuir en el desarrollo de competencias diferenciadoras que el contador público debe desarrollar y potenciar; además de dejar abierta la discusión sobre nuevas estrategias de competitividad como lo son las asociaciones de contadores públicos, de forma tal que se pueda ofrecer un servicio integrado y multidisciplinario para dar soluciones reales a un mercado cada vez más exigente en el aseguramiento de la información. 


\section{Propuesta de servicios de aseguramiento: consideraciones y guía}

Una propuesta de servicios profesionales, ya sea de revisoría fiscal o de auditoría externa, es la descripción y formalización del servicio profesional ofrecido, en donde se informa, entre otros, los términos contractuales, el alcance del servicio y los elementos diferenciadores que tiene el contador público o firma frente a sus colegas, para ser elegido como el revisor fiscal o auditor externo de un ente organizacional. La importancia de elaborar una adecuada propuesta de servicios, radica en que de ella depende en alto grado la contratación de los servicios profesionales, debido a que es el instrumento de estudio que tienen las asambleas de accionistas o juntas de socios en el caso del revisor fiscal, o de la alta administración en el caso de auditor externo, para tomar la decisión sobre la elección del mejor profesional o firma de profesionales para llevar a cabo el proceso de revisoría fiscal o auditoría externa en sus organizaciones.

A través de la propuesta de servicios de aseguramiento, las organizaciones obtienen criterios de selección para la contratación del revisor fiscal o auditor, mediante la comparación en términos de beneficio en el alcance del servicio propuesto y productos entregables versus su costo, es decir, el valor de los honorarios. Por lo tanto, la propuesta deberá ofrecer un servicio de calidad a cambio de un valor justo y competitivo de honorarios.

De acuerdo con lo anterior, la propuesta de servicios profesionales representa un factor crítico de éxito y es un elemento diferenciador para la obtención de clientes, además, permite al contador público analizar la rentabilidad del servicio que está ofreciendo; es decir, de cara al cliente, la propuesta refleja, en forma y en esencia, la calidad del servicio que se pretende prestar; y de cara al auditor, representa el alcance del trabajo y el margen de rentabilidad que se pretende obtener. Por lo tanto, la misma debe estar fundamentada en un adecuado conocimiento técnico de auditoría, del negocio, la industria y el sistema de costeo de los servicios ofrecidos, de tal forma que se presupueste correctamente la cantidad de horas de trabajo requeridas y la tarifa de la misma, evitando poner en riesgo la calidad del trabajo.

Los elementos básicos que deben conformar una propuesta de servicios profesionales de auditoría, son:

- Justificación

- Objetivos

- Alcance

- Enfoque y Metodología

- Equipo de trabajo

- Experiencia en el sector, industria o negocio 
- Productos entregables

- Honorarios

- Otras consideraciones

A continuación, se presentan las consideraciones para la elaboración de cada uno de los elementos mencionados.

\section{III.Justificación}

“... Tanto para la empresa objeto de auditoría como para el auditor, debe ser claro de dónde surge la necesidad de implementar un proceso de auditoría..."

Este primer elemento explica la necesidad de llevar a cabo el trabajo de auditoría en la organización. Dicha necesidad puede estar originada en el cumplimiento de alguna disposición legal o en las directrices del gobierno corporativo o administración de la compañía con algún objetivo específico. Con respecto a estos últimos, Cristina Aumatell (2003) indica que hay una latente necesidad por parte de los directivos, de reducir riesgos asociados a la gestión empresarial y a la organización en general, y de tratar la consolidación y generación de información como un área clave del negocio y no como una sección de soporte; es allí donde el aseguramiento de la misma juega un papel determinante para satisfacer dichas necesidades. Las crecientes necesidades de calidad en los reportes financieros, generadas tanto por usuarios internos como externos, son soportadas en los distintos niveles de aseguramiento de los informes emitidos por el revisor fiscal o auditor externo. Por lo tanto, se debe diferenciar cuándo las empresas requieren de la figura de revisor fiscal y cuándo la de un auditor externo, y especificar claramente cuando la elección de estos profesionales se hace en cumplimiento de las normas que así lo exijan o lo hacen de manera voluntaria.

En Colombia, la necesidad de tener revisor fiscal obedece a un aspecto de cumplimiento legal. De acuerdo con el artículo 13 de la Ley 43 de 1990, aquellas empresas con activos brutos iguales o superiores a 5.000 SMMLV o ingresos iguales o superiores a $3.000 \mathrm{SMMLV}$, están obligados a tener revisor fiscal (Congreso de Colombia, 1990). De igual forma, el artículo 203 del Código de Comercio obliga a todas las sociedades por acciones, las sucursales de compañías extranjeras y a las demás sociedades cuando su administración represente menos del 20\% de capital, a tener revisor fiscal (Presidencia de la República de Colombia, 1971).

Adicionalmente, el proceso de convergencia hacia prácticas contables y de aseguramiento de la información de aceptación internacional, iniciado con la Ley 1314 de 2009, derivó en la emisión del Decreto 0302 de 2015, el cual presenta el Marco Técnico Normativo de las Normas de Aseguramiento de la Información compuesto por el conjunto de normas emitidas por los diferentes organismos de la IFAC, entre ellas las Normas Internacionales de Auditoría 
Zamarra, J.; Guevara, J.; Ochoa, L. Lineamientos para elaborar una propuesta de servicios...

(NIA), que cobran gran relevancia al indicar el conjunto de procedimientos y requerimientos en relación con una auditoría de estados financieros.

Cabe destacar que el Marco Técnico Normativo de las Normas de Aseguramiento de la Información es de aplicación obligatoria para las entidades que, en Colombia, forman parte del Grupo 1 con base en lo dispuesto en el Decreto 2784 de 2012; y para aquellas que conforman el Grupo 2 con base en el Decreto 3022 de 2013, que adicionalmente cuenten con activos por un valor superior a 30.000 salarios mínimos mensuales legales vigentes (SMMLV) o que tengan más de 200 trabajadores. De igual forma, deben aplicar el Marco Técnico Normativo los revisores fiscales que dictaminen estados financieros consolidados de estas entidades (Ministerio de Comercio, Industria y Turismo, 2015).

La adopción de la normativa internacional supone un reto para los contadores públicos que actúan como revisores fiscales en el país. Su incorporación requiere cambios en la profesión contable, exigiendo del auditor una mejora en su desempeño, su competencia y cualificación para alcanzar los niveles de calidad exigidos por la normativa, lo que permite afrontar el cambio y a su vez disminuir el riesgo para la profesión (Rodríguez, García, y Ruiz, 2016). Lo anterior cobra mayor relevancia si se considera adicionalmente el desconocimiento de la normativa de obligatorio cumplimiento en el país, aun así, la convergencia a estándares internacionales brinda una oportunidad para la prestación de servicios a nivel internacional por parte de los contadores públicos que ejercen como auditores o revisores fiscales (Barreto y Marchena, 2016).

Es variada la legislación sobre la obligatoriedad de tener revisor fiscal en algunos sectores específicos de la economía, como son los fondos mutuos de inversión, cámaras de comercio, cajas de compensación familiar, asociaciones de autores, asociaciones gremiales, fondos de empleados, cooperativas, entre otros.

Lo anterior genera una dinámica en donde algunas empresas que contratan al revisor fiscal puede ser solamente para dar cumplimiento a la ley, por ello, se puede entender que algunos gerentes o administradores de las organizaciones, lo interpretan como un sinónimo de gasto, asumiendo la labor de esta figura como un conjunto de tareas rutinarias de revisión de saldos que no considera aspectos estructurales, que impiden percibir el verdadero valor que genera el ejercicio de la revisoría fiscal en una organización (Bustamante, 2008).

Esta situación impone un reto a los contadores que ejercen la revisoría fiscal, y es dar a conocer en principio, a través de la propuesta de servicios y luego con la ejecución de su trabajo, la condición de ser una figura independiente que aporta valor al aseguramiento y razonabilidad de la información financiera, la evaluación de lo adecuado del sistema de control 
interno y del cumplimiento legal; además de proporcionar diferentes niveles de seguridad mediante los informes de control que emite, brindando una contribución social a través de la responsabilidad de dar fe pública. Así mismo, debe considerarse la necesidad del mercado de contar con auditores y consultores con la experiencia suficiente para afrontar y guiar la aplicación de la normativa internacional, en pro de la eficiencia de los procesos y los resultados de la organización (Leuro, 2015), más aún en el caso de las empresas de menor tamaño que de forma voluntaria u obligatoria han comenzado a aplicar dicha normativa.

En el caso de la auditoría externa, la necesidad de las organizaciones de contar con esta figura, generalmente surge de los códigos de buen gobierno, o de algún requerimiento administrativo o de los socios o accionistas de evaluar algún proceso organizacional específico con algún propósito especial, por ejemplo, un fenómeno societario (fusión, escisión) o una reestructuración de procesos; o en el caso de que la organización cotice en alguna bolsa de valores del exterior, donde se requiere que sus estados financieros estén evaluados por un auditor externo, figura que opera a nivel internacional de manera generalizada.

De acuerdo con lo anterior, el elemento de justificación podrá variar dependiendo si la propuesta de servicios corresponde a una revisoría fiscal o a una auditoría externa. En conclusión, este elemento expone la importancia de llevar a cabo la auditoría, es decir, indica la necesidad de la organización que quedará cubierta con el servicio y soporta el siguiente elemento de la propuesta, que corresponde a los objetivos que persigue, evidenciando a la vez, los beneficios que se obtendrán.

Claves para la elaboración de la Justificación de un servicio profesional de Revisoría Fiscal o Auditoría externa:

En esta sección se debe reforzar el conocimiento de la compañía a auditar, dependiendo si es una entidad dedicada a la prestación de servicios o la elaboración de productos, si cuenta con una participación importante en el mercado colombiano, y la calidad y fortalecimiento de la marca.

De acuerdo con lo anterior, la compañía requiere el servicio de un auditor externo (profesional independiente o firma de auditores) de amplia y reconocida experiencia en la industria que le permita tener una adecuada identificación de los riesgos y oportunidades en sus procesos operacionales y financieros, garantizando no interferir con los principios éticos y de independencia que se deben tener en la relación de auditor y empresa auditada.

La firma de auditoría debe indicar los recursos con los que cuenta, el conocimiento y la experiencia necesaria para prestar un servicio de revisoría fiscal que cumpla con las funciones legales asignadas a esta figura, que agregue 
Zamarra, J.; Guevara, J.; Ochoa, L. Lineamientos para elaborar una propuesta de servicios...

valor y brinde confianza a los accionistas, a la administración y a los grupos de interés en general.

El servicio profesional ofrecido permitirá a la Compañía:

- Contar con un equipo de profesionales de diferentes áreas, contabilidad, impuestos, costos, tecnología, entre otras; con el suficiente conocimiento del negocio y de la industria, de tal forma que contribuyan al cumplimiento de los objetivos organizacionales en alineación con la estrategia corporativa; y que presenten un acompañamiento permanente en temas complejos de contabilidad.

- Contar con procesos de evaluación sobre lo adecuado del sistema de control interno, la razonabilidad de los estados financieros y el cumplimiento legal.

- Obtener diferentes informes de control, tales como certificaciones, informes de recomendaciones de valor que permitan corregir o mejorar los procesos organizacionales, además del dictamen de los estados financieros.

Tip: el elemento de justificación debe resaltar la necesidad que tiene la organización de elegirlo como revisor fiscal o auditor externo, por ello se recomienda asociar la necesidad de la organización con el valor agregado o factor diferenciador que posea quien está presentando la propuesta de servicios.

\section{Objetivo}

“... Tanto para la empresa objeto de auditoría como para el auditor, debe ser claro el tipo de servicio a prestar..."

En este ítem se plasma el objetivo del servicio, es decir, se plantea el propósito a alcanzar a través de la ejecución de las actividades inherentes a la revisoría fiscal. Este elemento es fundamental y encabeza el proceso de planeación del servicio, y le deja claro al ente objeto de auditoría, cuál será su resultado final. El objetivo varía dependiendo del trabajo de aseguramiento que requiera el cliente.

Con la implementación de normas internacionales de auditoría se amplía el abanico de servicios en la prestación de servicios profesionales por parte del contador público. Así:

- Trabajos de aseguramiento de la información financiera histórica (NIA 200810).

- Encargos de revisión de estados financieros (NITR 2400).

- Revisión de información financiera intermedia realizada por el auditor independiente de la entidad (NITR 2410).

- Trabajos para Atestiguar distintos de auditorías o revisiones de información financiera histórica (ISAE 3000). 
- Examen de información financiera prospectiva (ISAE 3400).

- Examen de los controles de las organizaciones de servicios (ISAE 3402).

- Encargos para realizar procedimientos acordados sobre información financiera (NISR 4400).

- Encargos de compilación de estados financieros (NISR 4410).

De esta forma el objetivo blinda ante el cliente y ante terceros el trabajo que se va a desarrollar y por ende las responsabilidades que asume el profesional contable, así es como se empiezan a delinear los distintos tipos de auditoría a desarrollar, como auditoría financiera, legal o de cumplimiento, operacional y administrativa (Blanco, 2012).

\section{Alcance}

“... La operación del día a día es responsabilidad de la administración... La opinión sobre la razonabilidad de la información financiera es responsabilidad del auditor..."

A través de este elemento, el revisor fiscal o auditor comunica de forma clara y precisa el trabajo que llevará a cabo; al igual que el anterior elemento, el alcance también depende del tipo de auditoría a realizar; y además incluye el periodo de tiempo cubierto por el trabajo de auditoría.

Para establecer el alcance, es fundamental tener claridad sobre:

- Tipo de auditoría a realizar.

- Responsabilidad de la administración vs responsabilidad del auditor.

- Normatividad aplicable al auditor.

- Conocimiento del ente organizacional, la normatividad que le aplica, sus procesos financieros y operacionales, además del sector o industria en la cual se desempeña.

Tipo de auditoría a realizar

El tipo de auditoría a realizar lleva implícita la connotación de los procesos que deberán ser auditados. Cada tipo de auditoría tiene un alcance sobre diferentes procesos de la organización, es así como a nivel de revisoría fiscal el alcance cubre el proceso de información financiera, el sistema de control interno y el cumplimiento regulatorio; en la auditoría externa el alcance normalmente esta sobre el proceso de información financiera y el sistema de control interno; en la auditoría financiera el alcance se enfoca en la evaluación de la razonabilidad de los estados financieros libres de error o fraude; en la auditoría de control interno sobre el sistema de control interno; en la auditoría operacional sobre los procesos operativos o ciclos de negocio; en la auditoría administrativa sobre la estructura de gestión (objetivos, planes, programas, procedimientos, controles) de la administración.

Responsabilidad de la administración vs responsabilidad del auditor

La responsabilidad de la administración y la del auditor, de manera general, se deriva de las siguientes disposiciones reglamentarias. Según el artículo 41 
de la ley 43 de 1990, en donde el revisor fiscal no puede ser responsable de los actos de la administración, el cual incluye la preparación y presentación de los estados financieros; mientras que según el artículo 7 de la Ley 43 de 1990 - Normas de auditoría generalmente aceptadas, el auditor es responsable de planear técnicamente la auditoría, ejercer una supervisión apropiada sobre los asistentes, evaluar el sistema de control interno de manera que se pueda confiar en él como base para la determinación de la extensión y oportunidad de los procedimientos de auditoría; y obtener evidencia válida y suficiente con el propósito de allegar bases razonables para el otorgamiento de un dictamen sobre los estados financieros sujetos a la revisión.

Para las figuras de revisoría fiscal y de auditoría externa, se encuentran otras responsabilidades específicas derivadas de normatividad local en el caso de la revisoría fiscal y de normatividad internacional (NIA - Normas Internacionales de Auditoría) en el caso de la auditoría externa.

A nivel de revisoría fiscal, se tiene que según el artículo 207 del Código de Comercio, las funciones del revisor fiscal son entre otras:

- Cerciorarse que las operaciones que se celebren o cumplan por cuenta de la sociedad se ajustan a las prescripciones de los estatutos, a las decisiones de la asamblea general y de la junta directiva.

- Dar oportuna cuenta, por escrito, a la asamblea o junta de socios, a la junta directiva o gerente, según los casos, de las irregularidades que ocurran en el funcionamiento de la sociedad y en el desarrollo de sus negocios.

- Colaborar con las entidades gubernamentales que ejerzan la inspección y vigilancia de las compañías, y rendirles los informes a que haya lugar o le sean solicitados.

- Velar por que se lleve regularmente la contabilidad de las sociedades y las actas de las reuniones de la asamblea, de la junta de socios y de la junta directiva, y porque se conserven debidamente la correspondencia de la sociedad y los comprobantes de las cuentas.

- Inspeccionar asiduamente los bienes de la sociedad y procurar que se tomen oportunamente las medidas de conservación o seguridad de los mismos y de los que ella tenga en custodia a cualquier otro título.

- Impartir instrucciones, practicar las inspecciones y solicitar los informes que sean necesarios para establecer un control permanente sobre los valores de la sociales.

- Convocar a la asamblea, junta de socios a reuniones extraordinarias cuando lo juzgue necesario.

- Cumplir las demás atribuciones que le señalen las leyes o los estatutos y las que, siendo compatibles con las anteriores, le encomiende la asamblea o junta de socios (Presidencia de la República de Colombia, 1971). 
En conjunto, el artículo 207 y 209 del Código de Comercio, contienen funciones que hacen responsable al revisor fiscal de llevar a cabo la auditoría de control y operacional (controles sobre los procesos operativos), financiera (razonabilidad de los estados financieros) y de cumplimiento legal de la normatividad aplicable.

A nivel de auditoría externa, bajo el contexto internacional, la NIA 700 establece que será responsabilidad del auditor expresar una opinión sobre los estados financieros basados en su auditoría, adicionalmente el informe debe indicar que el auditor cumple con los requisitos de ética así como que planifique y ejecute la auditoría con el fin de obtener una seguridad razonable sobre si los estados financieros están libres de errores materiales (International Auditing and Assurance Standards Board - IAASB, 2009).

Tip: De acuerdo con lo anterior, la propuesta de servicios deberá dejar en forma concisa la delimitación del alcance del trabajo en consideración tanto de las responsabilidades de la administración como del auditor. Los límites de una llegan hasta donde inician las funciones y responsabilidades de la otra; es precisamente esta consideración la que garantiza independencia y evita conflictos de intereses en las decisiones del ente organizacional.

\section{Normatividad aplicable al auditor y al ente objeto de auditoría}

El Marco Normativo basado en el decreto compilatorio 2420 de 2015, se basa en el marco contable de NIIF y de NIAS. Este cambio normativo, afecta actualmente el alcance del trabajo de los auditores. Ahora que las NIIF están en aplicación plena, los auditores deben dominar la aplicación de estas normas, pues no se puede auditar lo que no se conoce. Es así como el proceso de convergencia impuso el reto de capacitación a los profesionales que ejercen tanto la revisoría fiscal como la auditoría externa.

Aquí es necesario aclarar nuevamente que la responsabilidad de elaborar los estados financieros, sea bajo normas locales o bajo NIIF, es de la administración, según el artículo 41 de Ley 43, el revisor fiscal no será responsable de los actos administrativos relacionados con la elaboración de los estados financieros, el establecimiento de los procesos de control interno y la implementación de medidas anti fraude. Adicionalmente lo anterior involucra que el revisor fiscal no está obligado a rendir informes adicionales a los solicitados por la ley o que no sean de su competencia.

De acuerdo con la NIA 250 - Consideración de leyes y reglamentos en una auditoría de estados financieros, es responsabilidad de la administración asegurar que las operaciones de la entidad se conducen de acuerdo con las leyes y reglamentos. La responsabilidad por la prevención y detección de incumplimiento descansa en la administración. El auditor no es, y no puede ser considerado, responsable de prevenir el incumplimiento. El hecho de que se lleve a cabo una auditoría anual puede, sin embargo, actuar como una 
fuerza disuasiva o freno. Finalmente, la NIA "Objetivo y Principios Generales que Gobiernan una Auditoría de Estados Financieros”, el auditor deberá planear y desempeñar la auditoría con una actitud de escepticismo profesional reconociendo que la auditoría puede revelar condiciones o eventos que llevarían a cuestionarse si una entidad está cumpliendo con leyes y reglamentos.

Conocimiento del ente organizacional, la normatividad que le aplica, sus procesos financieros y operativos, además del sector o industria en la cual se desempeña

Parte del éxito de la propuesta de servicios consiste en el nivel de detalle con que se pueda estudiar el ente objeto de auditoría, dado que permite diseñar la propuesta con un alcance que se ajustará a la naturaleza y necesidades de la organización. Obtener este conocimiento implica una inversión de tiempo importante y en algunos casos puede llegar a ser complejo, sin embargo, es importante hacerlo, ya que proporciona elementos de valor para el diseño de la propuesta y el alcance del servicio que se ofrece, convirtiéndose en un diferencial para ser seleccionado como revisor fiscal o auditor.

Para delimitar correctamente el alcance del trabajo se debe conocer el objeto social de la organización a ser auditada y la industria en la que ésta se desempeña, de tal forma que se identifique su ente regulador (tipo de superintendencia) y la normatividad general y específica que le aplica, en cuanto a requerimientos de información financiera, de controles y sobre todo de requerimientos legales tales como certificaciones, transmisión de formatos o reportes o tipo de declaraciones tributarias. Tal como lo expresa la NIA 315, el conocimiento de la entidad es un factor fundamental para identificar los riesgos a los cuales está expuesta la entidad, que son específicos de un sector debido a su naturaleza y regulación. Y es con esos riesgos identificados donde se debe evaluar la extensión y calidad de los servicios a ofrecer.

Igualmente, es importante realizar pruebas analíticas para identificar los principales rubros y operaciones complejas y de estimados contables, entre otros que permitan planear recursos para cubrir las necesidades de la empresa a auditar.

Finalmente, la parte de la propuesta donde se plasma el alcance, debe incluir: 1) los acuerdos de la información que la empresa debe suministrar al revisor fiscal para el desarrollo del trabajo de auditoría, indicando que la entrega deberá ser oportuna y completa previa solicitud por escrito de parte del mismo revisor fiscal; 2) un párrafo que informe a la entidad que en el proceso de cierre de auditoría se requerirá una carta denominada carta de representación, la cual es firmada por el contador público y representante legal de la entidad y que contiene algunas manifestaciones sobre hechos económicos y sobre la veracidad de la información entregada el revisor 
fiscal; y 3) los entregables del auditor, que generalmente son memorandos de recomendaciones, informes de resultados de las diferentes fases de auditoría, certificaciones, declaraciones y la opinión final.

Tip: las principales fuentes de información para determinar un alcance de trabajo suficiente y adecuado son estados financieros comparativos, dictamen del anterior revisor fiscal, certificado de existencia y representación legal, posicionamiento en el mercado, referencias comerciales del ente organizacional y su junta directiva y normatividad aplicable.

\section{VI.Enfoque y Metodología}

"... La técnica es indispensable para lograr la excelencia..."

El enfoque se refiere a la forma en que el auditor orienta su trabajo. Normalmente, los trabajos de auditoría se componen de cuatro fases que permiten ejecutar las evaluaciones de forma ordenada y eficiente, así:

- Planeación: La planeación es la fase previa a la ejecución del trabajo de auditoría, que permite recopilar información útil para enfocar el trabajo en las áreas más importantes, guiar la ejecución de la auditoría, asignar el trabajo a los miembros del equipo de auditoría y promover el control de actividades; además, es aquí donde se anticipan los recursos a utilizar, entre ellos, el personal, el tiempo, los espacios, los materiales, y otros necesarios para llevar a cabo la auditoría (Montilla Galvis y Herrera Marchena, 2006).

Es entonces en este momento, donde a partir de un estudio detallado del ente organizacional se permite, diseñar un método de trabajo, conformar un equipo de profesionales calificado para atender el alcance del trabajo, identificar riesgos (inherentes, de control, de fraude, de auditoría), establecer la materialidad y finalmente conformar el PGA - Plan General de Auditoría (qué se va a auditar) y los programas de auditoría necesarios para llevarlo a cabo (cómo auditar, cuándo y a través de cuáles procedimientos). Por lo tanto, estos elementos deben ser evidenciados en la propuesta del auditor.

- Evaluación de los procesos: Es la fase en la que se ejecuta la evaluación de los flujos significativos de transacción en cuanto al componente de control interno, a través de pruebas de control. Estas pruebas de control son las que permiten que haya evidencia suficiente y apropiada sobre si los controles están diseñados, implementados y operando eficazmente, con el fin de integrar dentro del alcance de auditoría la evaluación de las deficiencias de control identificadas; es por esto, que se le recomienda al auditor revisar los informes emitidos por auditoría interna, que se refieran a los controles de información financiera, para identificar dichas deficiencias (Mantilla, 2013). 
Zamarra, J.; Guevara, J.; Ochoa, L. Lineamientos para elaborar una propuesta de servicios...

Es por esto, que el resultado de esta evaluación permite conocer si se debe aumentar o disminuir el alcance de las pruebas sustantivas que se realizan en la siguiente fase; además de permitir concluir sobre lo adecuado del sistema de control interno. En este punto se puede mencionar las metodologías, tipos de pruebas y muestreo a utilizar para las diferentes componentes del control interno.

- Procedimientos sustantivos: Como se mencionó anteriormente, su alcance depende de los resultados de la eficacia operativa del control interno, dado que cuando los controles son efectivos, disminuye la probabilidad de error y/o fraude en las cifras de los estados financieros, y por ende se reduce la necesidad de realizar procedimientos sustantivos. Según Arens, Elder, y Beasley (2007), con estos procedimientos se busca probar o verificar errores materiales en los estados financieros, entendiéndose dentro de estos procedimientos las pruebas sustantivas de operaciones, los procedimientos analíticos y las pruebas de detalle de saldos, los cuales se ejecutan en dos cortes, uno interino y otro de cierre de fin de año.

- Cierre de auditoría: Como lo expresa Lattuca (2008), la finalización de la auditoría implica la realización de varios procedimientos que complementen el juicio del auditor sobre la razonabilidad de los estados financieros, antes de concluir con el trabajo y de plasmar la opinión en el informe de auditoría. En el caso de la revisoría fiscal, auditoría externa o auditoría financiera, estas actividades incluyen la revisión de pasivos contingentes, de eventos posteriores, revisión de papeles de trabajo, resumen de resultados de las pruebas de control y sustantivas, comunicaciones con el comité de auditoría interna y la administración, solicitud de la carta de representación, determinación del tipo de dictamen a emitir; todo ello bajo el aseguramiento de la calidad del trabajo de auditoría con los diferentes niveles de supervisión.

Muchos auditores suelen seguir los procesos objeto de revisión, basados en modelos o normas reconocidas a nivel internacional, entre las que se destacan:

- Normas de auditoría para la planeación (NIA 300).

- Modelo COSO para la evaluación del Sistema de control interno de los procesos.

- Modelo COBIT para la evaluación del proceso de tecnología.

- Enfoque basado en riesgo para evaluación de procesos organizacionales significativos.

- Normas de Auditoría de Estados Financieros para el proceso contable y financiero.

A nivel internacional, y como parte de los elementos de aseguramiento, la mayoría de firmas de auditoría, estas son, tal y como se mencionó anteriormente, aquellas que auditen empresas clasificadas dentro del Grupo 
1 o dentro del Grupo 2 que posean activos por un valor superior a 30.000 SMMLV o que tengan más de 200 trabajadores, deben aplicar la normatividad relacionada con control de calidad NICC-1 y el código de ética, ambas normas son emitidas por la IFAC (Ministerio de Comercio, Industria y Turismo, 2015), la cual da confianza a los usuarios externos que el proceso y la aplicación de las metodologías de auditoría han sido revisadas y correctamente aplicadas.

En conclusión, en este ítem se debe resumir todo el conocimiento y la experiencia técnica acerca de cómo realizar un trabajo de auditoría de alta calidad y que cumpla a cabalidad las expectativas del cliente.

\section{Equipo de trabajo}

“... El talento humano como elemento diferenciador..."

Para Abdullah Y Adnan Ali Ertuğrul (2015), en una auditoría, un factor importante para la efectividad de los resultados, es contar con un equipo de trabajo, debido a la complejidad de las estructuras y los procesos en las empresas auditadas, a los exigentes requisitos técnicos y a la latente necesidad de contar con diferentes puntos de vista sobre situaciones específicas, que serían muy subjetivas y complejas de analizar con la presencia de un solo auditor. Asegura, además, que una auditoría es más eficiente si el auditor deja a un lado el hábito de trabajar en documentos, e invierte buena parte de su tiempo a tener presencia física en el proceso a auditar (on-site audit), con el fin de obtener toda la información de primera mano que esté a su alcance, y para esto, se requiere dividir el trabajo en varios auditores competentes, debido a la gran cantidad de procesos y dificultad que esto implica.

Por otra parte, el artículo 211 del código de comercio establece que "El revisor fiscal responderá de los perjuicios que ocasione a la sociedad, a sus asociados $\mathrm{o}$ a terceros, por negligencia o dolo en el cumplimiento de sus funciones" (Presidencia de la República de Colombia, 1971).

De acuerdo con lo anterior, es necesario, que el revisor fiscal o auditor, cuente con el conocimiento suficiente, individual o en conjunto con su equipo de trabajo, para presentar un servicio de calidad según la naturaleza de la organización y para cumplir con la Ley 43 de 1990 que regula el ejercicio del contador público, además con las normas de auditoría.

Claro está que, aunque al realizar la auditoría con equipo de trabajo se garantiza una mayor objetividad y eficiencia en la ejecución de la auditoría, el costo asociado es mucho mayor, al que se incurre con un solo auditor. Por lo tanto, como lo dice Solomon (1982), se debe evaluar el costo-beneficio en la determinación de un equipo de trabajo y de sus integrantes, ya que hay clientes en los que no se requiere mucha presencia de personal, por sus sencillas estructuras y procesos. 
Zamarra, J.; Guevara, J.; Ochoa, L. Lineamientos para elaborar una propuesta de servicios...

Si se trata de una firma de auditoría, en este aparte de la propuesta, se expone una reseña de la misma, su misión, visión, valores, estructura de trabajo, servicios interdisciplinarios, experiencia en el sector, nivel de formación de sus profesionales, entre otros.

Dentro de los equipos de trabajo es igualmente importante describir el personal de expertos o especialistas que apoyarán las labores del revisor fiscal o auditor, y que son claves al momento de rendir informes con valor agregado para la compañía ya que poseen conocimientos técnicos específicos. La elección de estos especialistas dependerá de las necesidades que se identifiquen en el momento de hacer el estudio de la empresa objeto de auditoría; normalmente se incluyen especialistas en materia de impuestos, sistemas de información, finanzas, costos y en algunos casos en derecho.

Tip: como elemento diferenciador en este ítem se pude incluir todo lo relacionado con responsabilidad social corporativa que realicen las firmas de auditoría como parte de su modelo de negocios.

\section{Experiencia en el sector, industria o negocio}

“... Para auditar es necesario conocer lo que se audita..."

Con este elemento, se pretende demostrar la experiencia de la firma o del auditor en organizaciones similares a aquella a la cual se está presentando la propuesta de servicios profesionales. Es por esto, que incluso en muchas ocasiones, suele anexarse certificados que expiden aquellas empresas sobre la prestación del servicio por parte del auditor y la satisfacción frente a la calidad del mismo.

Esta información proporciona confiabilidad frente a la competencia del auditor para llevar a cabo el servicio de auditoría en una organización con características particulares al sector, industria o negocio al que pertenezca, de tal forma que se demuestre conocimiento que contribuya a un servicio de calidad.

En vista de la creciente complejidad de las estructuras y procesos de los negocios, hoy en día los auditores se están especializando en una industria específica, buscando desarrollar y adquirir conocimientos más profundos sobre el sector en el que se especializan; generando así mayor eficacia y eficiencia en la auditoría. Este conocimiento adquirido incide tanto en la naturaleza y alcance de la auditoría como en las evaluaciones de riesgos realizadas desde la planificación; ya que a mayor conocimiento específico de la industria, de mayor calidad será la evaluación de riesgos de la auditoría (Low, 2004).

Tener experiencia en un sector específico facilita la precisión en los resultados de la auditoría. Se resalta el caso de China que, en el 2012, reguló que los socios extranjeros debían ser menos del $20 \%$ en las oficinas de los 4 Big para el 2017. Como consecuencia de esta regulación, se ha generado un 
aumento significativo de la participación de los auditores chinos en las oficinas, y se ha ganado mayor experiencia en el mercado, siendo poco probable que disminuya la calidad de auditoría a largo plazo (Cahan y Sun, 2015).

Complementario a esto, hay que destacar que no sólo se debe relacionar la experiencia y la especialización con la calidad de la auditoría, sino que también se debe relacionar la proporción de clientes que se auditan. De acuerdo con Cahan, Jeter, y Naiker (2011) los honorarios de auditoría y la calidad son más altos cuando el auditor es especialista en un sector, y gana una cuota de mercado significativa al auditar una baja proporción de clientes en esa industria.

Hay muchas discusiones sobre si realmente la experiencia va directamente relacionada con la calidad de la auditoría, pero tal como lo expone Bonner (1990), estas diferencias en opiniones o resultados de investigaciones, se pueden dar porque algunos de esos estudios no consideran el papel del conocimiento específico en los auditores, ignorando la especialización del sector como factor fundamental al evaluar la incidencia de la experiencia en la calidad de la auditoría.

Los conocimientos específicos sobre un sector particular de la industria incluyen requerimientos legales o de cumplimiento; adicionalmente cada sector de la economía tiene unos riesgos inherentes que deberán ser identificados desde la propuesta para incluir su cobertura dentro de los programas de auditoría.

Las características personales de los auditores principales, entre estas la experiencia, se toman como indicios sobre la calidad de la auditoría que se va a realizar. Una de las habilidades con las que debe contar todo auditor, es la capacidad de crítica haciendo referencia al escepticismo profesional, y esta crítica solo será acertada si se tienen bases, y por supuesto experiencia sobre lo que se audita. En referencia a esto, Farmer, Rittenberg, y Trompeter (1987) argumenta que los auditores con mayor experiencia tienden a estar más en desacuerdo sobre el tratamiento contable preferido por el cliente que los auditores inexpertos.

Los auditores experimentados presentan mayor coherencia en la selección de información relevante y la respuesta en la evaluación de los riesgos, emitiendo juicios en menos tiempo y más acertados. Esto se debe a que la experiencia afecta la exactitud de los juicios de los auditores, ya que cuando los auditores emiten sus juicios, necesitan extraer la información necesaria contenida en su memoria y relacionarla entre sí para llegar a una precisión que va a depender de la cantidad de información que se tenga en la cabeza sobre la situación a auditar (Bonner, 1990).

En esta sección de la propuesta, se podría decir que en general las firmas de auditoría tienen mayor ventaja frente a los auditores independientes, dado que 
las primeras tienen la infraestructura suficiente para formar o tener auditores especializados por industria que acumulan un gran bagaje de conocimientos muy específicos al trabajar en diferentes clientes del mismo sector. El auditor independiente puede optar por especializarse en uno o dos sectores de la economía si quiere competir frente a una firma.

Al concluirse que los auditores con más experiencia tienen un mayor conocimiento en el trabajo, tienen juicios más precisos y por ende sus auditorías tiene mejor calidad que la de los auditores poco experimentados; es un ítem que no se puede dejar por alto en la propuesta de servicios profesionales, ya que los clientes están dispuestos a reconocer dicho conocimiento y calidad con mayores honorarios y esto representará una ventaja competitiva también a la hora de ser seleccionada la propuesta de auditoría.

\section{IX.Productos entregables}

“... Los resultados deben ser comunicados clara y oportunamente..."

Los informes a entregar, en el caso del revisor fiscal deben estar soportados en la normatividad legal, especialmente en lo indicado en los artículos 207, 208 y 209 del Código de Comercio. En el caso de los auditores corresponden a lo indicado en las normas de auditoría y en los acuerdos específicos que se hagan con la administración. Los informes más usuales son:

- Informes de recomendaciones: contienen los hallazgos observados sobre los procesos auditados junto con las recomendaciones para mejorar o corregirlos y el beneficio de su implementación.

- Dictamen de estados financieros: es el resultado final del trabajo del revisor fiscal o auditor financiero y dependerá de los resultados de la auditoría operacional (pruebas de controles), de cumplimiento legal y financiera (pruebas sustantivas).

En este punto se resalta la responsabilidad que tendrá el auditor de hacer referencia a las Cuestiones Clave de Auditoría (KAM, por sus siglas en inglés) dentro de su informe de auditoría, tal y como lo expresa el Decreto 2170 de 2017 en su artículo 5, en consonancia con lo establecido en el Decreto 2420 de 2015, modificado a su vez por el Decreto 2132 de 2016 (Ministerio de Comercio, Industruia y Turismo, 2017). Los KAM tienen como objetivo "mejorar el valor comunicativo del informe de auditoría al proporcionar una mayor transparencia acerca de la auditoría que se ha realizado" (International Auditing and Assurance Standards Board - IAASB, 2015), de esta forma el auditor deberá plasmar en su dictamen información adicional en relación con aquellas cuestiones de mayor relevancia presentadas a lo largo de la ejecución de la auditoría, dicha información le permitirá al usuario de la información tener un mayor acercamiento con la realidad de la organización, como un complemento 
de la opinión del auditor que determina si la información financiera presentada por la entidad es o no razonable.

- Declaraciones tributarias y certificaciones: vienen dadas por ley, se presentan de acuerdo a la industria y sector específico al que pertenezca la empresa auditada. En Colombia existen sectores de la economía altamente regulados que exigen al revisor fiscal un nivel de vigilancia adicional, con requerimientos de cumplimiento propios de ese sector, ejemplo el sector financiero o el sector salud.

Tip: es importante que una vez se sea elegido como revisor fiscal o auditor se emita una declaración o carta de compromiso donde se establezca varios de los elementos de la propuesta de auditoría, tales como alcance, equipo de trabajo, forma y entrega de información requerida, honorarios, etc., en señal de aceptación por parte del ente organizacional; y que en la misma, se indique que los papeles de trabajo son de propiedad exclusiva del revisor fiscal o auditor y constituyen información confidencial y reservada que será archivada por el oferente de acuerdo las políticas y procedimientos del mismo. Lo anterior se basa en el artículo 9 de la Ley 43 de 1990.

La denotación clara y concisa de los anteriores productos a entregares muy importante, para dejar constancia que cualquier producto adicional a entregar, no descrito en esta propuesta, estará sujeto a una ampliación de los honorarios descritos más adelante. Este ítem reviste una especial importancia ya que es común que las empresas soliciten la realización de informes distintos derivadas de hechos pasados, presentes y sobre todo futuros como por ejemplo la evaluación de nuevas leyes o requerimientos de información, procesos de auditoría relacionados con futuras fusiones, combinaciones de negocios o escisiones o requerimiento adicionales producidos por la firma de la empresa de convenios futuros por ejemplo la necesidad que el revisor fiscal emita certificaciones productos de un convenio privado y no por producto de una certificación originada por la ley.

Tip: en esta sección de la propuesta el revisor fiscal o auditor puede incluir aquellos informes que está dispuesto a entregar como valor agregado.

\section{Honorarios}

“... La coherencia entre lo que se hace y lo que se cobra, contribuye a un trabajo de calidad..."

Como se expuso en la parte inicial de este artículo, los honorarios se convierten en un elemento importante de diferenciación de la propuesta de servicios y por ello los mismos se deben establecer con criterios tanto técnicos como comerciales.

Enfatizando en la parte técnica, la razonabilidad de los honorarios se analiza teniendo en cuenta los criterios de la calidad de los servicios requeridos. En 
Zamarra, J.; Guevara, J.; Ochoa, L. Lineamientos para elaborar una propuesta de servicios...

cuanto a esto, el AICPA (2015) menciona que la contratación de un auditor que carece de conocimientos de la naturaleza de la industria específica y de las habilidades necesarias para realizar las auditorías, está en contravía con el objetivo de proteger al cliente; es por esto que sólo después de evaluar las características técnicas del auditor, se deben revisar los precios ofrecidos por los auditores clasificados.

Los honorarios de auditoría están asociados positivamente con el tamaño, el riesgo y la complejidad del cliente. Variables que representan el nivel de esfuerzo al que se verá sometido el auditor. Siendo el tamaño, por sí sola, una variable fundamental y crítica para cualquier modelo de honorarios de auditoría, ya que por el tamaño se deduce que tiene buena capacidad financiera para responder a sus obligaciones, siendo ésta una de tantas razones por la cual se evalúa este ítem (Hay, Knechel, y Wong, 2006).

Para la cuantificación de los honorarios se debe involucrar un conocimiento previo de la entidad el cual debe tener ciertos niveles de rigurosidad. Algunos elementos claves a tener en cuenta son:

- Riesgos asociados a las operaciones que realiza la compañía y a las cuentas o rubros asociados al control interno.

- La complejidad de los sistemas de información de la compañía.

- La regulación aplicable en materia de certificaciones y declaraciones de acuerdo al sector económico que se desempeñe la compañía.

- Ubicación geográfica de la compañía incluyendo su dirección general, oficinas, sucursales, filiales sujetas a consolidación o visitas de revisión.

- Estatutos de la compañía.

- Niveles preliminares de la calidad del control interno de la compañía incluido el tono de la gerencia en cuento al control interno del reporte de información financiera.

- Calidad y experiencia tanto por parte de la administración como del equipo de auditoría asignado.

- Concepción general de las pruebas de auditoría a realizar y posibles escenarios de muestreo para un cálculo preliminar de las horas a invertir en el trabajo de auditoría.

- Identificación de temas los cuales deben ser desarrollados y auditados por expertos.

- Posibilidades de la utilización del trabajo de otros como auditores internos o auditores de calidad de la misma compañía.

- Identificación de organizaciones de servicios (outsourcing) y como afectan los mismos el alcance del trabajo de auditoría.

Todos estos factores permiten estimar el número de horas de trabajo necesarias a invertir para el cumplimiento y desarrollo a plenitud de todas las obligaciones del revisor fiscal o auditor. En Colombia, ni la cantidad de horas 
por tipo de revisión ni el valor de la hora de trabajo del auditor están reguladas, por lo tanto, dependerá del nivel de experiencia del equipo de auditoría y de las condiciones de mercado.

La fijación de los honorarios es una tarea delicada, en el cual la ética profesional y el interés de la auditoría no permiten que los valores presupuestados sean muy altos o muy bajos. Adicionalmente, como la evaluación de riesgos de las cuentas contables y procesos relevantes, se produce después de la contratación, una mala estimación de horas puede generar daños en el auditor, ya que, aunque los honorarios adicionales pueden ser negociados, el cobro no está garantizado.

Una vez realizado el cálculo de las horas, es necesario asignarles el valor de las tarifas de los ejecutores, supervisores y especialistas que deben intervenir. Para así hallar el valor mínimo de los honorarios a cobrar de tal efecto que no se incurran en pérdidas por parte del revisor fiscal o auditor, y a su vez se asegure que se cumplan con las funciones.

Por otra parte, hay que tener en cuenta, una vez más, la experiencia del auditor en la determinación de los honorarios, no sólo por el hecho de que a mayor experiencia, mayor calidad de auditoría y por ende mayores honorarios, sino también porque según Castro, Peleias, y Silva (2015) si la auditoría se va a realizar por primera vez en un cliente y/o sector, los honorarios tienden a reducirse, ya que los auditores buscan cobrar menos en el primer año para recuperarse en los años siguientes.

Claro está que debe existir un balance entre los honorarios estimados y la oferta del mercado, ya que una de las razones de los clientes para cambiar de auditor es por la oferta de otra firma o auditor de un precio inferior. El hecho de que ofrezcan precios más bajos, no significa necesariamente que la calidad de la auditoría sea menor, puede ser, como lo expresa Hay et al. (2006) "las comisiones más bajas pueden deberse a que las empresas de auditoría ofrezcan intencionalmente servicios con descuento para ganar nuevos negocios ("lowballing") o porque un nuevo auditor puede ofrecer un servicio más eficiente, justificando una reducción de tarifas" (p.176).

En algunas ocasiones y sobre todo cuando se espera conservar relaciones comerciales de mediano plazo es común dar un porcentaje de "descuento" el cual debería ser recuperado en fechas futuras y son básicamente las horas empleadas del conocimiento del negocio y de la industria que en vigencias futuras no serían necesarias aplicarlas en su totalidad.

Las implicaciones de proponer un honorario elevado son varias, de acuerdo con Lin y Hwang (2010), altos honorarios pagados al auditor, aumentan el vínculo económico entre el auditor y el cliente y, por lo tanto, pueden perjudicar la independencia del auditor. Con una independencia deteriorada, la 
Zamarra, J.; Guevara, J.; Ochoa, L. Lineamientos para elaborar una propuesta de servicios...

calidad de la auditoría se torna a ser dudosa, ya que se aumentaría la gestión de los ingresos.

Ahora, esa gestión de resultados se podría evitar si la comisión de auditoría es de calidad, y consecuentemente, la auditoría en general se tornará de mejor calidad. Es por esto que, dentro de los elementos a evaluar, se debe analizar el papel de los comités de auditoría de los clientes.

Primero, hay que establecer cómo son determinados los honorarios de los auditores pertenecientes a los comités de auditorías y luego ver la incidencia de éstos en los honorarios del auditor externo o revisor fiscal. De acuerdo con Zaman, Hudaib, y Haniffa (2011), los honorarios de las auditorías internas, están positivamente alineadas con la eficacia de las mismas, la regularidad con que la junta de la empresa se reúna con las mismas, la escasez de dualidad de roles, el tamaño del cliente, la complejidad de la estructura y el sector al que pertenezca. En lo referente a esto Abbass y Aleqab (2013) complementan que las características de los auditores internos ayudan a incrementar la confianza de los auditores externos en sus trabajos, traduciéndose en un menor alcance de auditoría, minimizando los esfuerzos de los auditores externos y por ende las tarifas. En la presentación de los honorarios es muy importante agregar el respectivo valor del IVA, así como la forma de pago por parte del cliente, lo usual es que este pago se realice mes vencido a partir del momento de la posesión del revisor fiscal o auditor.

Es importante mencionar que la retribución económica se convierte en un derecho fundamental del revisor fiscal o auditor regulado en la Ley 43, artículo 46:

Siendo la retribución económica de los servicios profesionales un derecho, el contador público fijará sus honorarios de conformidad con su capacidad científica o técnica y en relación con la importancia y circunstancia en cada uno de los casos que le corresponda cumplir, pero siempre previo acuerdo por escrito entre contador público y el usuario.

A su vez el artículo 59 de la Ley 43 de 1990, ratifica el proceso de negociación de honorarios en donde indica:

"En los concursos para la prestación de servicios profesionales de un contador público o de sociedades de contadores, es legítima la competencia en la medida en que la adjudicación se deba a la calidad de los servicios del oferente. No será legítima ni leal cuando la adjudicación obedezca a reducciones posteriores al valor cotizado originalmente o al ofrecimiento gratuito de servicios adicionales a los cotizados".

Desafortunadamente, en muchas ocasiones, el valor de los honorarios que están dispuestos a pagar los entes objeto de auditoría, no compensan la cantidad de trabajo necesario para cumplir con las funciones del revisor fiscal, generando pérdidas o baja rentabilidad para el mismo, y en el peor de los casos 
haciendo que éste asuma riesgos en el proceso de auditoría con un alcance insuficiente, deteriorando el servicio prestado y la imagen de la revisoría fiscal haciendo a su vez que las empresas no estén dispuestas a pagar el valor justo de los honorarios del revisor fiscal.

No sobra contextualizar el suceso de convergencia a IFRS en Colombia, ya que, debido a esto, el costo de los revisores fiscales o auditores incrementó en gran medida por la actualización de conocimientos en NIAS y NIIF. En el caso de China, en los primeros años de adopción de las NIIF se observó un aumento significativo en los honorarios de auditoría de empresas nacionales y más aún en empresas internacionales de auditoría, por esperarse un mejor servicio en vista de que estas últimas tienen más experiencia con el manejo de la norma (Lin y Ai-Ru, 2016). Es por esto que se menciona como un periodo de transición que representa una gran oportunidad para incrementar los honorarios, haciendo alusión al esfuerzo que implica la actualización a NIIF.

Como ya se mencionó anteriormente el monto de los honorarios siempre será un factor importante de decisión para la empresa al momento de elegir al revisor fiscal o auditor.

\section{XI.Otras consideraciones}

- Cláusula de confidencialidad: en algunos casos y específicamente cuando en las propuestas se incluyen elementos importantes de la metodología producto del trabajo intelectual del oferente de la propuesta, o de los temas incluidos como valor agregado, es importante incluir una cláusula de confidencialidad que indique explícitamente que lo mencionado en la propuesta no podrá ser utilizado ni divulgado a otros contadores públicos oferentes ni por la misma empresa en sus procesos internos.

- Recursos de trabajo provistos por el ente auditado: el revisor fiscal o auditor debe realizar la solicitud formal de los recursos que requiere para el desarrollo de sus funciones, y que van por cuenta del ente auditado, tales como un espacio físico donde pueda desempeñar sus funciones en forma independiente; los gastos de viaje, desplazamientos, hoteles, gastos secretariales, entre otro; el acceso de consulta a las aplicaciones tecnológicas de la empresa auditada, necesario para la realización del proceso de auditoría.

- Recursos de trabajo provistos por el auditor: el auditor debe contar con herramientas tecnológicas de auditoría entre los que se pueden destacar repositorios electrónicos de papeles de trabajo; formas automáticas para la generación de memorandos de planeación, de programas de auditoría tanto globales como detallados, de planillas sumarias y sub sumarias; software especializado en la evaluación de bases de datos que incluyan procesos estadísticos para la realización de muestreo por saldos 
Zamarra, J.; Guevara, J.; Ochoa, L. Lineamientos para elaborar una propuesta de servicios...

o software especializado para la realización de pruebas puntuales de auditoría como por ejemplo la valoración de inversiones negociables o de realización de cálculos detallado de depreciaciones o de cartera por metodologías de pérdida esperada, entre otros. Este tipo de herramientas definitivamente dan valor agregado a los clientes y ahorran muchas horas de trabajo de auditoría pudiéndose invertir un mayor número de horas en análisis y en el cumplimiento a cabalidad de las funciones del revisor fiscal o auditor externo.

- En algunas compañías es común que adicional a la propuesta de servicios profesionales, soliciten una exposición a que debe ser realizada por los distintos oferentes con unos tiempos y condiciones especificadas. Usualmente esta exposición se realiza con la ayuda de una presentación que sintetice los principales elementos de la propuesta.

- Tipo de contratación: el tipo de contratación del revisor fiscal debe asegurar total independencia. La Circular Externa 115000011 de la Superintendencia de Sociedades permite un modelo de contratación por prestación de servicios o inclusive permite un modelo de contrato laboral con algunos requisitos establecidos por la Ley 50 de 1990 (Superintendencia de Sociedades, 2008). Este modelo de contrato laboral, aunque es permitido por la ley es evidente que va en contra de la total independencia del revisor fiscal. En la práctica el modelo que se observa es el denominado carta de compromiso, la cual se deriva en gran medida de la propuesta de auditoría, en este documento se informan las responsabilidades del revisor fiscal, los productos a entregar por el mismo y de las obligaciones por parte de las compañías en cuanto al pago convenido de los honorarios junto con algunas condiciones como por ejemplo: la entrega a tiempo de la información requerida por el revisor fiscal para la ejecución de sus actividades, este documento debe ir firmado por el representante legal de la compañía y por el propio revisor fiscal.

- Para el caso del auditor, si se trata de una auditoría externa sobre la razonabilidad de los estados financieros, también se deberá asegurar total independencia. En otros casos de auditorías específicas contratadas directamente por la administración y no por los socios o accionistas, no existe restricción en la forma de contratación.

\section{Conclusiones}

La propuesta es el elemento de interacción entre los oferentes y las compañías que necesitan de los servicios de revisoría fiscal o de auditoría externa y los contadores públicos, la elaboración de una propuesta clara y completa que cumpla con unos requisitos mínimos por parte de la firma o revisor fiscal, permitirá a la empresa que tomará la decisión contar con una 
base sólida con la que pueda soportar su elección considerando aspectos relacionados con su necesidad específica así como la relación costo beneficio del servicio ofertado por los proponentes. Así mismo, la propuesta se convierte en el medio idóneo para que el oferente presente de una forma atractiva el servicio que puede prestarle al cliente, atendiendo a su capacidad operativa y de conocimiento en la industria.

Una propuesta con una presentación adecuada que contenga todos los elementos que suministren a la Compañía suficiente información que dé cuenta de un trabajo de alta calidad, puede ser elemento diferenciador para ser elegido como auditor externo o revisor fiscal de una compañía.

El establecimiento de los honorarios debe ser hecho con criterios técnicos basados en las horas a invertir en el proceso de auditoría para poder cumplir a satisfacción con todas las responsabilidades del revisor fiscal y que adicionalmente no genere pérdidas para el contador público que ejecute dichas funciones.

Con el objeto de ofrecer servicios integrados que realmente cubran las necesidades de las empresas, las firmas de contadores públicos deben buscar socios con diferentes especializaciones e inclusive diferentes disciplinas, que cubran necesidades de auditoría en áreas como la tributaria, control, finanzas, sistemas de información computarizada y legales.

Como futuras líneas de investigación derivados de las reflexiones planteadas en el presente artículo podrían efectuarse investigaciones asociadas con el análisis sobre variables de las propuestas de auditoría como los honorarios, horas de dedicación, cantidad de integrantes de los equipos de trabajo y cualificación de los líderes de los compromisos, reconociendo que para el caso colombiano existen muchas limitaciones al abordar este tipo de estudios ya que la inmensa mayoría de las compañías no revelan las propuestas entregadas por los auditores de las cuales puede tomarse la información requerida para este tipo de estudios.

\section{Referencias bibliográficas}

Abbass, D. A., \& Aleqab, M. M. (2013). Internal auditors' characteristics and audit fees: evidence from egyptian firms. International Business Research, 67-80.

Abdullah, A., \& Adnan Ali Ertuğrul, Y. (2015). The impact of average duration of experience, duration of on-site audit and team work on magniture of errors detected in financial statements: an application of financial audit of public administrations by the TCA. Journal of Turkish Court of Accounts / Sayistay Dergisi, 107-127.

Accountants, T. A. (2015). Obtaining Quality Employee Benefit Plan Audit Services: The Request for Proposal and Auditor Evaluation Process. Recuperado el 09 de Julio de 2017, de AICPA : https://www.aicpa.org/InterestAreas/EmployeeBenefitPlanAuditQuality/ Resources/PlanSponsorResourceCenter/DownloadableDocuments/Plan_Sponsor_ Guidelines_preparing_RFP.pdf 
Zamarra, J.; Guevara, J.; Ochoa, L. Lineamientos para elaborar una propuesta de servicios...

Arens, A., Elder, R., \& Beasley, M. (2007). Auditoría, un enfoque integral. México: Pearson educación.

Aumatell, C. S. (2003). La auditoría de la información, componente clave de la gestión estratégica de la información. El profesional de la información, 261-268.

Barreto, B. E., \& Marchena, M. (2016). Incidencia del nuevo marco normativo de auditoría en el ejercicio de la revisoría fiscal en Colombia. Dictámen libre, 18, 23-29.

Blanco, Y. (2012). Auditoría Integral, normas y procedimientos. Bogotá: Ediciones ECOE.

Bonner, S. E. (1990). Experience Effects in Auditing: The Role of Task-Specific Knowledge. The Accounting Review, 72-92.

Bustamante , H. C. (2008). Los imaginarios del revisor fiscal: una aproximación teórica. Contaduría Universidad de Antioquia, 155-198.

Cahan , S. F., \& Sun, J. (2015). The effect of audit experience on audit fees and audit quality . Journal of Accounting, Auditing \& Finance, 78-100.

Cahan, S. F., Jeter, D. C., \& Naiker, V. (2011). Are all industry specialist auditors the same? AUDITING: A journal of practice \& theory, 191-222.

Castro, W. B., Peleias, I. R., \& Silva, G. P. (2015). Determinantes dos honorários de auditoría: um estudo nas empresas listadas na bm\&fbovespa, Brasil. Revista contabilidade \& finanças, 261-173.

Congreso de Colombia. (13 de Diciembre de 1990). Ley 43 de 1990. Diario Oficial No. 39602. Bogotá, Colombia. Obtenido de https://www.mineducacion.gov.co/1759/ articles-104547_archivo_pdf.pdf

Farmer, T. A., Rittenberg, L. E., \& Trompeter, G. M. (1987). An investigation of the impact of economic and organizational factors on audit independence. Auditing: A journal of practice \& theory, $1-14$.

Hay, D. C., Knechel, R., \& Wong, N. (2006). Audit fees: a meta-analysis of the effect of supply and demand attributes. Contemporary Accounting Research, 141-191.

International Auditing and Assurance Standards Board - IAASB. (15 de Diciembre de 2009). International Standards on Auditing 700 Forming an opinion and reporting on financial statements. Obtenido de International Federation of Accountants: http:// www.ifac.org/system/files/downloads/a036-2010-iaasb-handbook-isa-700.pdf

International Auditing and Assurance Standards Board - IAASB. (Enero de 2015). International Standard on Auditing 701. Communicating Key Audit Matters in the independing auditor's report. Obtenido de IAASB - Auditing \& Assurance | IFAC: https:/www.iaasb.org/system/files/publications/files/ISA-701_2.pdf

Lattuca, A. J. (2008). Compendio de auditoría. Buenos Aires: Temas grupo editorial.

Leuro, A. P. (2015). Convergencia de normas internacionales de auditoría y aseguramiento en las Pymes colombianas y su relación con los riesgos asumidos por los auditores. Tendencias, 17(1), 142-153.

Lin, H.-L., \& Ai-Ru, Y. (2016). The Effects of IFRS Adoption on Audit Fees for Listed Companies in China. Asian Review of Accounting, 43-68.

Lin, J. W., \& Hwang, M. I. (2010). Audit quality, corporate governance, and earnings management: a meta-analysis. International Journal of Auditing, 57-77. 
Low, K.-y. (2004). The Effects of Industry Specialization on Audit Risk Assessments and Audit-PlanningDecisions. The Accounting Review, 201-219.

Mantilla, S. A. (2013). Auditoría de control interno. Bogotá: Ecoe Ediciones.

Ministerio de Comercio, Industria y Turismo. (20 de Febrero de 2015). Decreto Número 0302 de 2015. Diario Oficial, Edición 49.431, 12-245. Colombia. Obtenido de http://www.comunidadcontable.com/BancoMedios/Documentos\%20PDF/d-030215(mincomercio).pdf

Ministerio de Comercio, Industruia y Turismo. (22 de Diciembre de 2017). Decreto 2170 de 2017. Obtenido de Presidencia de la República: http://es.presidencia.gov. co/normativa/normativa/DECRETO\%202170\%20DEL\%2022\%20DE\%20DICIEMBRE\%20 DE\%202017.pdf

Montilla Galvis, O., \& Herrera Marchena, L. G. (2006). El deber ser de la auditoría. Estudios Gerenciales, 83-110.

Presidencia de la República de Colombia. (16 de Junio de 1971). Código de Comercio. Diario Oficial No. 33.339. Bogotá, Colombia. Obtenido de http://www.secretariasenado.gov. co/senado/basedoc/codigo_comercio.html

Presidencia de la República de Colombia. (16 de Junio de 1971). Decreto 410 de 1971, Código de Comercio. Diario Oficial No. 33.339. Colombia.

Rodríguez, D. M., García, C. A., \& Ruiz, J. C. (2016). La auditoría y su control de calidad: una mirada desde las normas de aseguramiento de la información en Colombia. Contexto, 5, 63-74.

Solomon, I. (1982). Probability Assessment By Individual Auditors And Audit Teams - An Empirical-Investigation. Journal of Accounting Research, 689-710.

Superintendencia de Sociedades. (21 de Octubre de 2008). Circular externa 115-000011. Obtenido de Superintendencia de Sociedades: https:/www.supersociedades.gov.co/ nuestra_entidad/normatividad/normatividad_circulares/28996.pdf

Zaman, M., Hudaib, M., \& Haniffa, R. (2011). Corporate governance quality, audit fees and non-audit services fees. Journal of Business Finance \& Accounting, 165-197. 